\title{
Molecular Structural Dynamics Captured with Ultrafast Electron Diffraction
}

\section{Martin Centurion}

\author{
University of Nebraska, Lincoln, Nebraska, United States
}

The conversion of light into chemical and mechanical energy is the first step in many processes in nature such as photosynthesis, vision, DNA photodamage, where this conversion takes place at the level of single molecules. The light absorption and ensuing structural dynamics are also relevant for solar energy conversion and storage by molecules. In order to understand and control these processes, it is essential to be able to observe them, ideally mapping the position of the atoms in the molecule as a function of time, i.e. making a molecular movie. This requires a measurement tool that simultaneously reaches subAngstrom resolution in space and femtosecond resolution in time, to capture the structural dynamics on its natural spatial and temporal scales. These kind of measurements have recently become possible through the use of femtosecond electron pulses in pump-probe diffraction experiments where the molecules are excited by a laser pulse and probed by the scattering of a short electron pulse with a well-defined time delay. In our work we have focused on studying the dynamics of isolated molecules (in the gas phase) in order to observe the intrinsic response of the molecules, free of external interactions. Studying isolated molecules also provide a good benchmark for comparing with theory.

Recent developments in ultrafast electrons sources, in particular the use of relativistic (MeV) electron pulses for gas phase UED, have allowed us to reach a temporal resolution approaching $100 \mathrm{fs,} \mathrm{while} \mathrm{at}$ the same time determining interatomic distances with precision below 0.1 Angstrom. With relativistic electron sources, shorter electron pulses can be delivered on the sample due to the reduced Coulomb broadening, and the experiment does not suffer from the temporal blurring produced by the velocity mismatch between laser and electrons [1]. We have performed a series of experiments at the SLAC MeV UED facility where we have first demonstrated the required spatiotemporal capability by capturing coherent vibrational motion in excited iodine molecules [2]. Since then, we have captured the dynamics in more complex reactions were we have observed the splitting of the nuclear wavepacket as it traverses a conical intersection [3], determined the structure of short-lived intermediate states and observed coherent nuclear motions that persist after the molecules return to the ground state [4]. Through recent improvements in the instrument, we now expect to be able to capture dynamics in a large class of molecular reactions [5].

\section{References}

1. J. Yang et al, "Femtosecond gas phase electron diffraction with MeV electrons" Faraday Discussions 194, 563 (2016).

2. J. Yang et al, "Diffractive Imaging of Coherent Nuclear Motion in Isolated Molecules" Physical Review Letters, 117, 153002 (2016).

3. J. Yang et al, "Imaging CF3I conical intersection and photodissociation dynamics with ultrafast electron diffraction" Science 361, 64 (2018) .

4. K. J. Wilkin et al, "Diffractive imaging of dissociation and ground-state dynamics in a complex molecule" Phys. Rev. A 100, 023402(2019).

5. X. Shen et al, "Femtosecond gas-phase mega-electron-volt ultrafast electron diffraction" Structural Dynamics 6, 054305 (2019). 